\title{
Future of Computing Science
}

\author{
R K SHYAMASUNDAR \\ IIT Bombay
}

\section{If people do not believe that mathematics is simple, \\ It is only because they do not realize how complicated life is.}

John von Neumann, 1947.

Computing has made a huge impact on science, society and even national security. We have reached a point, wherein significant progress either in science or society is dependent on the computing power. To mention a few of the areas where computing has made a huge impact is: smart materials, understanding structures, earthquake engineering, epidemiology, genomics, molecular modeling, chemistry, astronomy, biology, e-commerce, e-governance, health-care, disaster management, national security, and public infrastructures.

Computer Science is undergoing a fundamental change and is reshaping our understanding of the world. An important aspect of this change is the theory and applications dealing with the gathering and analyzing of large real-world data sets. In this section, we introduce four research projects in which processing and interpreting large data sets is a central focus. Innovative ways of analyzing such data sets allow us to extract useful information that we would never have obtained from small or synthetic data sets, thus providing us with new insights into the real world.

Modern Computer Science is undergoing a fundamental change. In the early years of the field, computer scientists were primarily concerned with the size, efficiency and reliability of computers. They attempted to increase the computational speed as well as reduce the physical size of computers, to make them more practical and useful. The research mainly dealt with hardware, programming languages, compilers, operating systems and databases. Meanwhile, theoretical computer science developed an underlying mathematical foundation to support this research, which in turn, led to the creation of automata theory, formal languages, computability, and algorithm analysis. Through the efforts of these researchers, computers have shrunk from the size of a room to that of a dime, nearly every modern household has access to the Internet and communications across the globe are virtually instantaneous.

Computers can be found everywhere, from satellites hundreds of miles above us to pacemakers inside beating human hearts. The prevalence of computers, together with communication devices and data storage devices, has made vast quantities of data accessible. This data incorporates important information that reveals a closer approximation of the real world and is fundamentally different from what can be extracted from individual entities. Rather than analyzing and interpreting individual messages, we are more interested in understanding the complete set of information from a collective perspective. However, these large-scale data sets are usually far greater than the data sets that can be processed by traditional means. Thus, future computer science research and applications will be less concerned with how to make computers work and more focused on the processing and analysis of such large amounts of data.

Various strategies and specific takeaways have been discussed in various chapters in articulating a Roadmap of investment on ICT.

Looking at the impact computing has been

\footnotetext{
*Authorfor Correspondence: E-mail: shyamasundar@gmail.com ${ }^{1}$ IEEE COMSOC 2020 Report, June 2012.

${ }^{2}$ Zhiwei Xu and Guojie Li, Computing for the Masses, Communications of the ACM, 54, 10, pp. 129-137
} 
making along with possible disruptions in science, technology and society, the following simple takeaways as captured in the book from Microsoft titled. "The Fourth Paradigm: Data Intensive Science Discovery" provide simple advice that need to be adhered by one and all to carry the benefits of computing to mankind:
If you are a scientist, talk to a computer scientist about your challenges, and vice versa.

If you are a student, take classes in both science and computer science.

If you are a teacher, mentor, or parent, encourage those in your care toward interdisciplinary study in addition to giving them the option to specialize. 